\title{
Future directions in the treatment of $B K$ virus nephropathy
}

\author{
Wisit Cheungpasitporn $^{1^{*}}$, Charat Thongprayoon ${ }^{2}$, Supavit Chesdachai ${ }^{3,4}$, Jackrapong Bruminhent $^{4}$ \\ ${ }^{1}$ Department of Internal Medicine, Division of Nephrology, University of Mississippi Medical Center, Jackson, Mississippi, USA \\ ${ }^{2}$ Department of Internal Medicine, Bassett Medical Center, Cooperstown, NY, USA \\ ${ }^{3}$ Department of Medicine, University of Minnesota, Minnesota, USA \\ ${ }^{4}$ Department of Medicine, Faculty of Medicine Ramathibodi Hospital, Mahidol University, Thailand
}

\section{A R T I C L E I N F O}

\section{Article Type:}

Editorial

\section{Article History:}

Received: 14 April 2017

Accepted: 23 August 2017

ePublished: 30 November 2017

\section{Keywords:}

BK virus

Treatment

Kidney transplantation

Polyoma virus

Transplantation

\begin{abstract}
Implication for health policy/practice/research/medical education:
BK polyomavirus-associated nephropathy (BKVAN) is an important cause of allograft renal dysfunction after kidney transplantation. Recent evidence has suggested associations of BK infection with urological malignancies and the development of de novo donor specific antibody (DSA). Despite recent advances in kidney transplantation knowledges, management of BKVAN has remained challenges. Recent evidence has suggested that BK-specific T-cell responses, but not neutralizing antibodies, is important in clearance of BK viremia. In this article, we present future directions in prevention and treatment of BKVAN.
\end{abstract}

Please cite this paper as: Cheungpasitporn W, Thongprayoon C, Chesdachai S, Bruminhent J. Future directions in the treatment of BK virus nephropathy. J Nephropharmacol. 2018;7(1):3-5. DOI: 10.15171/npj.2018.02.
$\mathrm{B}$ K polyomavirus-associated nephropathy (BKVAN) is an important cause of allograft renal dysfunction after kidney transplantation (1), up to $10 \%$ of kidney transplant patients $(2,3)$. In addition, recent evidence has suggested associations of BK infection with urological malignancies (4) and the development of de novo donor specific antibody (DSA) $(5,6)$, resulting in antibody mediated rejection and allograft loss.

Although a few screening methods for BK virus have been applied, transplanted kidney biopsy is still the gold standard for diagnosis of BKVAN $(7,8)$ and intranuclear viral inclusions-SV40 positive staining (or in situ hybridization for BK virus) is pathognomonic $(8,9)$. However, SV40- negative inflammation with viremia can be found in $22.8 \%$ of cases, prior to subsequent pathological confirmation of BKVAN, which could be due to 1) missed disease from sampling error or 2) viral reactivation following $\mathrm{T}$ cell rejection (10). This ongoing issue causes challenges and difficulties in diagnosis and management of BKVAN.

\section{Current treatments of BKVAN}

Despite recent advances in kidney transplantation knowledges, management of BKVAN has remained challenges. Although a few medications that have been proposed and used as adjunctive therapies for BKVAN (Table 1), clinical benefits of these adjunctive therapies are currently controversial. Thus, careful immunosuppression reduction/modification remains the mainstay treatment for BKVAN (11). However, reducing immunosuppression may put patients at risks for rejection (3). Moreover, as mentioned prior, SV40-negative inflammation with viremia can often be found leading to the difficulties in differentiation in diagnosis of BKVAN, rejection, or concurrent rejection and BKVAN. This ongoing problem results in significant use of bolus corticosteroids in this setting. However, recent evidence suggested deleterious effects of bolus corticosteroids on a higher rate of graft loss in patients with BK infection (12). Brincidofovir, a novel agent for treatment of BK virus infection (Table 1), has been developed to reduce adverse effect and increase the efficacy of cidofovir $(13,14)$. Some case reports/case series demonstrated its efficacy for treatment of $\operatorname{BKVAN}(13,14)$. However, the recent data on brincidofovir for treatment of cytomegalovirus (CMV) infection unfortunately showed that brincidofovir was not found to be more effective than placebo, and the rate of CMV infection was higher after drug discontinuation $(15,16)$. In addition, brincidofovir also has worrisome gastrointestinal side effects, mimicking graft-versus-host disease (GVHD) (17). 
Table 1. Proposed Adjuvant therapies for BKVAN

\begin{tabular}{lll}
\hline Adjuvant therapies & In vitro activity & Clinical benefit for BK infection \\
\hline Cidofovir & Yes & Yes/No (case reports, retrospective studies, meta-analysis) (11); + Cost-effective (26) \\
Brincidofovir & Yes & N/A (case reports, case series and phase II NCT00793598) (18) \\
Leflunomide & Yes & No (Clinical trials) (18) \\
Fluoroquinolone & Yes & No (clinical trials) $(27,28)$ \\
mTor inhibitors & Yes & No (retrospective studies) $(18)$ \\
Intravenous immunoglobulin & Yes & Yes (retrospective studies) $(18,20)$ \\
\hline
\end{tabular}

\section{Future directions}

In patients under immunosuppressive or immunomodulatory therapy including kidney transplant recipients, lacks of BK-specific $\mathrm{T}$ cell response and/or neutralizing antibodies have been proposed as underlying pathogenesis of high BK viral replication and cytopathic damage, leading to BKVAN development (18). In-vitro, intravenous immunoglobulin (IVIG) provided the inhibitory effect on BK virus replication via $\mathrm{BK}$ virus neutralizing antibodies (19). Despite a need of multicenter randomized trial confirming its clinical benefits, retrospective studies have shown potential benefits of combination treatment with reduced immunosuppression an IVIG on clearing BK virus infection (18-20).

Recent evidence has suggested that BK-specific T-cell responses, but not neutralizing antibodies, is important in clearance of BK viremia (21). Thus, T cell adoptive therapy has been proposed as potential novel treatment of BK infection (22) by transferring $\mathrm{T}$ cell that recognizes the $\mathrm{BK}$ virus antigen to BK virus-infected recipients. Very recently, Papadopoulou et al (22) transferred viral-specific $\mathrm{T}$ cell to 7 hematopoietic stem cell transplant recipients with BK viremia. Investigators report successful treatment response without significant serious adverse effects. Future studies of this novel adoptive T cell therapy for BK virus infection are required in kidney transplant setting.

\section{Prevention of BKVAN and individualization of BKVAN} risk

To date, a number of risk factors for BK virus infection have been proposed including donor-, recipient-, and transplant-associated risk factors, which some factors are modifiable $(3,23)$. Within recent years, pretransplantation donor-recipient pair seroreactivity against $\mathrm{BK}$ virus has been found to a strong predictor of BK viremia and BKVAN after kidney transplantation (24). Pre-transplantation BK serological testing of potential donors and recipients may help stratify patient risk for BK infection after kidney transplantation. Since different types of immunosuppression result in different risks for BK infection (25), future studies are required if the use of pre-transplantation BK serological testing of potential donors and recipients in prediction model for BKVAN development can help choose appropriate immunosuppression for each recipient in order to reduce BKVAN while weighing rejection risk.

\section{Authors' contribution}

All authors had access to the data and a role in writing the manuscript. All authors read and signed the final paper.

\section{Conflicts of interest}

The authors declare that they have no conflicting interest.

\section{Ethical considerations}

Ethical issues (including plagiarism, data fabrication, double publication) have been completely observed by the authors.

\section{Funding/Support}

None.

\section{References}

1. Lamarche C, Orio J, Collette S, Senecal L, Hebert MJ, Renoult E, et al. BK Polyomavirus and the Transplanted Kidney: Immunopathology and Therapeutic Approaches. Transplantation. 2016;100:2276-87. doi: 10.1097/ tp.0000000000001333.

2. Bohl DL, Brennan DC. BK virus nephropathy and kidney transplantation. Clin J Am Soc Nephrol. 2007;2 Suppl 1:S36-46. doi: 10.2215/cjn.00920207.

3. Sawinski D, Goral S. BK virus infection: an update on diagnosis and treatment. Nephrol Dial Transplant. 2015;30:209-17. doi: 10.1093/ndt/gfu023.

4. Papadimitriou JC, Randhawa P, Rinaldo CH, Drachenberg CB, Alexiev B, Hirsch HH. BK Polyomavirus Infection and Renourinary Tumorigenesis. Am J Transplant. 2016;16:398406. doi: 10.1111/ajt.13550.

5. Sawinski D, Forde KA, Trofe-Clark J, Patel P, Olivera B, Goral S, et al. Persistent BK viremia does not increase intermediate-term graft loss but is associated with de novo donor-specific antibodies. J Am Soc Nephrol. 2015;26:96675. doi: 10.1681/ASN.2014010119.

6. Cheungpasitporn W, Mitema D, Amer H, Cosio F, Stegall M, Schinstock C, editors. BK Nephropathy, but Not Isolated BK Viremia Associated with De Novo DSA Despite Similar Immunosuppression Reduction. Am J Transplant. 2016;16:357-357. doi: 10.1111/ajt.14161.

7. Kasiske BL, Zeier MG, Chapman JR, Craig JC, Ekberg H, Garvey CA, et al. KDIGO clinical practice guideline for the care of kidney transplant recipients: a summary. Kidney int. 2010;77:299-311. doi: 10.1038/ki.2009.377.

8. Masutani K, Shapiro R, Basu A, Tan H, Wijkstrom M, Randhawa P. The Banff 2009 Working Proposal for polyomavirus nephropathy: a critical evaluation of its utility as a determinant of clinical outcome. Am J Transplant. 
2012;12:907-18. doi: 10.1111/j.1600-6143.2012.03993.x.

9. Lusco MA, Fogo AB, Najafian B, Alpers CE. AJKD Atlas of Renal Pathology: Polyomavirus Nephropathy. Am J Kidney Dis. 2016;68:e37-e38. doi: 10.1053/j.ajkd.2016.10.007.

10. Nankivell BJ, Renthawa J, Sharma RN, Kable K, O’Connell PJ, Chapman JR. BK Virus Nephropathy: Histological Evolution by Sequential Pathology. Am J Transplant. 2017;17:2065-2077. doi: 10.1111/ajt.14292.

11. Johnston O, Jaswal D, Gill JS, Doucette S, Fergusson DA, Knoll GA. Treatment of polyomavirus infection in kidney transplant recipients: a systematic review. Transplantation. 2010;89:1057-70. doi: 10.1097/TP.0b013e3181d0e15e.

12. Fishman JA. Infection in Organ Transplantation. Am J Transplant. 2017;17:856-79. doi: 10.1111/ajt.14208.

13. Reisman L, Habib S, McClure GB, Latiolais LS, Vanchiere JA. Treatment of BK virus-associated nephropathy with CMX001 after kidney transplantation in a young child. Pediatric transplantation. 2014;18:E227-31. doi: 10.1111/ petr. 12340 .

14. Papanicolaou G, Kolitsopoulos Y, Young J, Boruchov A, Sankaranarayanan N, Hull D, et al. BK Virus-Associated Nephropathy (BKVN), an Under-Recognized Cause of Renal Dysfunction in Severely Immunosuppressed Hematopoietic Stem Cell Transplant (HSCT) Patients: Report of 5 Cases of Bkvn and the Potential Role of CMX001 for Treatment. Biology of Blood and Marrow Transplantation. 2013;19:S302-3. doi: 10.1016/j. bbmt.2012.11.442.

15. Marty F, Winston D, Chemaly R, Boeckh M, Mullane $\mathrm{K}$, Shore $\mathrm{T}$, et al. Brincidofovir for prevention of cytomegalovirus (CMV) after allogeneic hematopoietic cell transplantation (HCT) in CMV-seropositive patients: a randomized, double-blind, placebo-controlled, parallelgroup phase 3 trial. 2016;22(3):S23. doi: 10.1016/j. bbmt.2016.01.009.

16. El-Haddad D, El Chaer F, Vanichanan J, Shah DP, ArizaHeredia EJ, Mulanovich VE, et al. Brincidofovir (CMX001) for refractory and resistant CMV and HSV infections in immunocompromised cancer patients: A single-center experience. Antiviral research. 2016;134:58-62. doi: 10.1016/j.antiviral.2016.08.024.

17. Detweiler CJ, Sung AD, Saullo JL, Prasad VK, Cardona DM, editors. Brincidofovir (CMX001) Toxicity: Another Potential Mimicker of Gastrointestinal Graft Versus Host Disease. Laboratory Investigation. 2016:96:169A-169A.

18. Barth H, Solis M, Lepiller Q, Sueur C, Soulier E, Caillard S, et al. 45 years after the discovery of human polyomaviruses BK and JC: Time to speed up the understanding of associated diseases and treatment approaches. Crit Rev Microbiol.
2017;43:178-95. doi: 10.1080/1040841x.2016.1189873.

19. Randhawa PS, Schonder K, Shapiro R, Farasati N, Huang Y. Polyomavirus BK neutralizing activity in human immunoglobulin preparations. Transplantation. 2010;89:1462-5. doi: 10.1097/TP.0b013e3181daaaf1.

20. Kable K, Davies CD, O'Connell P J, Chapman JR, Nankivell BJ. Clearance of BK virus nephropathy by combination antiviral therapy with intravenous immunoglobulin. Transplant Direct. 2017;3:e142. doi: 10.1097/ txd.0000000000000641.

21. Leboeuf C, Wilk S, Achermann R, Binet I, Golshayan D, Hadaya K, et al. BK Polyomavirus-Specific 9mer CD8 T Cell Responses Correlate With Clearance of BK Viremia in Kidney Transplant Recipients: First Report From the Swiss Transplant Cohort Study. Am J Transplant. 2017. doi: 10.1111/ajt.14282.

22. Papadopoulou A, Gerdemann U, Katari UL, Tzannou I, Liu $\mathrm{H}$, Martinez C, et al. Activity of broad-spectrum T cells as treatment for AdV, EBV, CMV, BKV, and HHV6 infections after HSCT. Sci Transl Med. 2014;6:242ra83. doi: 10.1126/ scitranslmed.3008825.

23. Ambalathingal GR, Francis RS, Smyth MJ, Smith C, Khanna R. BK Polyomavirus: clinical aspects, immune regulation, and emerging therapies. Clin Microbiol Rev. 2017;30:50328. doi: 10.1128/cmr.00074-16.

24. Wunderink HF, van der Meijden E, van der Blij-de Brouwer CS, Mallat MJ, Haasnoot GW, van Zwet EW, et al. Pretransplantation donor-recipient pair seroreactivity against BK polyomavirus predicts viremia and nephropathy after kidney transplantation. Am J Transplant. 2017;17:16172. doi: 10.1111/ajt.13880.

25. KDIGO clinical practice guideline for the care of kidney transplant recipients. Am J Transplant. 2009;9 Suppl 3:S1155. doi: 10.1111/j.1600-6143.2009.02834.x.

26. Hua DK, Howard K, Craig JC, Chapman JR, Wong G. Cost-effectiveness of cidofovir treatment of polyomavirus nephropathy in kidney transplant recipients. Transplantation. 2012;93:188-94. doi: 10.1097/ TP.0b013e31823e7b0e.

27. Knoll GA, Humar A, Fergusson D, Johnston O, House AA, Kim SJ, et al. Levofloxacin for BK virus prophylaxis following kidney transplantation: a randomized clinical trial. JAMA. 2014;312:2106-14. doi: 10.1001/jama.2014.14721.

28. Lee BT, Gabardi S, Grafals M, Hofmann RM, Akalin E, Aljanabi A, et al. Efficacy of levofloxacin in the treatment of BK viremia: a multicenter, double-blinded, randomized, placebo-controlled trial. Clin J Am Soc Nephrol. 2014;9:583-9. doi: 10.2215/cjn.04230413.

Copyright $\odot 2018$ The Author(s); Published by Society of Diabetic Nephropathy Prevention. This is an open-access article distributed under the terms of the Creative Commons Attribution License (http://creativecommons.org/licenses/by/4.0), which permits unrestricted use, distribution, and reproduction in any medium, provided the original work is properly cited. 